\title{
Stability analysis for some numerical schemes of partial differential equation with extra measurements
}

\author{
Nazi Abdollahi (D), Davood Rostamy* (D) \\ Department of Mathematics, Imam Khomeini International University, Qazvin, Iran
}

\begin{abstract}
This paper is devoted to study the stability analysis of some finite difference schemes for an inverse problem with unknowns time-dependent coefficients subject to extra measurements. We prove that the popular forward time centered space scheme is a conditional method. But the backward time centered space and Crank Nicolson methods are suitable schemes because they are unconditional methods. We justify this advantage of the stability analysis versus the some numerical methods with an example. All the results and a numerical example are in two-dimensional setting.
\end{abstract}

Mathematics Subject Classification (2010). 65M12, 65M15, 65M60, 82D10, 35L80

Keywords. Von Neumann stability analysis, inverse problems, finite difference methods.

\section{Introduction}

Various types of physical and environmental phenomena such as heat, electrostatic, electrodynamics, fluid dynamics and pollution could be modelled in the form of partial differential equations (PDEs) $[1,2,19,20]$. Usually, in study of these phenomena all data of problem is available. However, for some practical problems, part of boundary data, or initial data, or diffusion coefficients, or source term may not be given and we have to find them by extra measurement data which will yield to some inverse problems like evolutionary inverse problems, boundary inverse problems, coefficient inverse problems and source inverse problems, respectively $[4,6,13,18,22,24,27]$.

In the past several decades, various numerical techniques have been developed to solve inverse problems $[3,10,14,15,21]$. In these problems extra measurement data are used to obtain unique solution. Extra measurement of some problems play an important role in mathematical science, physics and engineering. These problems are widely envisaged in the modeling of many physical phenomena, namely diffusion and conduction of natural materials [29].

In this paper, we consider the following coefficient inverse problem with variable coefficients in a general bounded domain.

\footnotetext{
*Corresponding Author.

Email addresses: abdollahin7@gmail.com (N. Abdollahi), rostamy@khayam.ut.ac.ir (D. Rostamy)

Received: 04.09.2016; Accepted: 03.03.2018
} 
Let $\Omega \subseteq \mathbb{R}^{2}$ be a bounded domain with sufficient smooth boundary $\partial \Omega$. The coefficient inverse problem we considered is to determine unknowns coefficients $\left(a_{j m}(x, t)\right)_{j, m=1}^{2}$ from the following equations

$$
\left\{\begin{array}{rlrl}
\frac{\partial u}{\partial t}(x, t)=\sum_{j, m=1}^{2} a_{j m}(x, t) \frac{\partial^{2} u(x, t)}{\partial x_{j} \partial x_{m}} & & \\
& +\varepsilon \cdot \nabla u(x, t)+d(t) u(x, t), & & x \in \Omega, \quad 0<t \leq T, \\
u(x, 0)=f(x), & & x \in \bar{\Omega}, \quad \\
u(x, t)=g(t), & x \in \partial \Omega, \quad 0 \leq t \leq T,
\end{array}\right.
$$

subject to extra measurements

$$
u\left(x^{*}, t\right)=E(t), \quad \nabla u\left(x^{*}, t\right)=F(t), \quad 0 \leq t \leq T,
$$

in which $x^{*} \in \Omega, d, f, g, E$ and $F$ are given functions and $\varepsilon$ is a constant function. We assume that $\left(a_{j m}(x, t)\right)_{j, m=1}^{2}$ are sufficiently smooth coefficients.

Certain types of physical problems can be modeled by (1.1) [11, 16, 28]. In recent decades, various numerical methods have been used to approximate the solution of spacial case of (1.1). In [6] the author reduced the problem to a nonlinear integral equation. This approach depends on the explicit form of the fundamental solution of the heat operator and does not easily extend to the higher dimensional cases. In [4] backward Euler finite difference method is applied. Also, the discrete version of the maximum principle for finite difference schemes is used to show the stability of method. The finite element method is applied by the authors of [7]. Dehghan [8] used several finite difference schemes for identifying the function $u(x, t)$ and unknown coefficient $a(t)$ in a one dimensional case of (1.1). It has been shown that these methods are efficient but have not been discussed about the stability of them.

In this paper, we generalized the problem in [8] and attempt to complete the work presented in that. We use three finite difference schemes, forward time centered space (FTCS), backward time centered space (BTCS) and Crank- Nicolson (CN) method for inverse problem (1.1). Then, we discuss about the stability of them.

Various methods have been constructed for the analysis of stability among them von Neumann stability analysis is the most common method used to determine the stability. This method is based on Fourier analysis therefore it is usually limited to linear PDEs with constant coefficients $[23,26]$. Although, the condition of linear equation with constant coefficients are not as restrictive as it might seem. In the case of the equation is nonlinear, we can apply linearization methods and transform it into a linear equation. When coefficients are not constant, we implement principle of frozen coefficients. According to this principle, we consider PDEs where coefficients do not change too rapidly. So, such coefficients can be considered to be almost constant at each point in space and time $[9,17]$.

This paper is organized as follow. In Section 2, we discuss about the von Neumann stability analysis for some PDEs with variable coefficients. Implementation of FTCS, BTCS and CN methods for inverse problem (1.1) and determine the stability region of these methods are presented in Section 3. In Section 4, we give a numerical example to illustrate our theoretical discussion. Finally, we give a conclusion in Section 5.

\section{Von Neumann stability analysis for linear parabolic PDEs with vari- able coefficients}

In this section, we explain how to apply von Neumann stability analysis as presented in [5] for linear partial differential equations (PDEs) with variable coefficients.

Consider the following problem: 


$$
\begin{cases}u_{t}(x, t)=a(x, t) u_{x x}(x, t), \quad x \in(0,1), & 0<t<T, \\ u(x, 0)=\varphi(x), & x \in[0,1], \\ u(0, t)=f(t), & 0 \leq t \leq T, \\ u(1, t)=g(t), & 0 \leq t \leq T,\end{cases}
$$

where $T$ is a positive constant, $\varphi(x), f(t), g(t)$ and $a(x, t)$ are known functions and $a(x, t)>$ 0 . In this problem $a(x, t)$ is not constant, so to implement the von Neumann stability analysis, we use the principle of frozen coefficients. Suppose

$$
Q_{T}=\{(x, t) \mid 0 \leq x \leq 1, \quad 0 \leq t \leq T\},
$$

is discretized uniformly into an $M \times N$ mesh with the spatial step size $h=\frac{1}{M}$ in $x$ direction and the time step size $k=\frac{T}{N}$, respectively. Replace the region $Q_{T}$ by a set of grid points $\left(x_{p}, t_{q}\right)$ are defined by

$$
\begin{cases}x_{p}=p h, & p=0,1, \cdots, M, \\ t_{q}=q k, & q=0,1, \cdots, N,\end{cases}
$$

in which $M$ and $N$ are integers. Assume that $\varphi_{p}, f^{q}, g^{q}$ and $u_{p}^{q}$ be the notations of finitedifference approximation of $\varphi\left(x_{p}\right), f\left(t_{q}\right), g\left(t_{q}\right)$ and $u\left(x_{p}, t_{q}\right)$, respectively.

We apply FTCS method to (2.1) (see [25]), so:

$$
\begin{cases}\frac{u_{p}^{q+1}-u_{p}^{q}}{k}=a(x, t) \frac{u_{p+1}^{q}-2 u_{p}^{q}+u_{p-1}^{q}}{h^{2}}, & p=1,2, \cdots, M-1, \\ & q=0,1,2, \cdots, N-1, \\ u_{p}^{0}=\varphi_{p}, & p=0,1,2, \cdots, M, \\ u_{0}^{q}=f^{q}, & q=0,1,2, \cdots, N, \\ u_{M}^{q}=g^{q}, & q=0,1,2, \cdots, N .\end{cases}
$$

We freeze the coefficient $a(x, t)$ of problem $(2.2)$, on the other hand we assume that $a(x, t)$ is fixed at each point of space and time. We apply the von Neumann stability analysis to find the stability region. Also, we assume that the solution is of the form

$$
u_{p}^{q}:=G^{q} e^{i \beta p h} .
$$

Substitution of the above expression into first equation of (2.2) yields

$$
G^{q+1} e^{i \beta p h}-G^{q} e^{i \beta p h}=\operatorname{ra}(x, t)\left[G^{q} e^{i \beta(p+1) h}-2 G^{q} e^{i \beta p h}+G^{q} e^{i \beta(p-1) h}\right] .
$$

Here $G=e^{i \alpha k}$ is the growth factor such that $-\pi<\alpha<\pi$ is the grid wave number and $r=\frac{k}{h^{2}}$. After simplifying equation (2.3), we have

$$
G-1=r a(x, t)\left[e^{i \beta h}-2+e^{-i \beta h}\right],
$$

therefore

$$
\begin{aligned}
G & =1+r a(x, t)[2 \cos (\beta h)-2] \\
& =1-4 r a(x, t) \sin ^{2}\left(\frac{\beta h}{2}\right) .
\end{aligned}
$$

The stability condition for $(2.2)$ is $|G| \leq 1$. Hence

$$
-1 \leq 1-4 r a(x, t) \sin ^{2}\left(\frac{\beta h}{2}\right) \leq 1,
$$

and

$$
r \leq \frac{1}{2 a(x, t) \sin ^{2}\left(\frac{\beta h}{2}\right)} .
$$


In order to place the strongest limitation on $r$, we have to choose $\beta h$ such that the right hand side of inequality be minimum. This minimum happens when $\sin ^{2}\left(\frac{\beta h}{2}\right)=1$. Therefore, we have

$$
r \leq \frac{1}{2 a(x, t)}
$$

The above relation was achieved by the principle of frozen coefficient [9].

Relation (2.4) can be interpreted in two different ways.

(i) If constant values of $k, h$ and hence $r$ are employed over the entire grid, then we have to ensure that

$$
r \leq \frac{1}{2 \max _{x, t} a(x, t)}
$$

(ii) If the time step $k$ changes, then at every time level, $k$ is to be chosen so that

$$
r(t) \leq \frac{1}{2 \max _{x} a(x, t)} .
$$

Let us consider the following problem

$$
\begin{cases}u_{t}(x, t)=a(x, t) u_{x x}(x, t)+b(x, t) u_{x}(x, t)+c(x, t) u(x, t), & x \in(0,1), \\ u(x, 0)=\varphi(x), & 0<t<T, \\ u(0, t)=f(t), & x \in[0,1], \\ u(1, t)=g(t), & 0 \leq t \leq T, \\ & 0 \leq t \leq T,\end{cases}
$$

where $T$ is a positive constant and $a, b, c, \varphi, f$ and $g$ are given functions, $a(x, t)>0$ and $c(x, t) \leq 0$ for $(0,1) \times(0, T)$. If each of $a:=a(x, t), b:=b(x, t)$ and $c:=c(x, t)$ are constant, then problem (2.5) has a solution by the form $u(x, t)=\varphi(x) \exp (c t)$.

By applying the FTCS method for (2.5), we have

$$
\begin{gathered}
\frac{u_{p}^{q+1}-u_{p}^{q}}{k}=a \frac{u_{p+1}^{q}-2 u_{p}^{q}+u_{p-1}^{q}}{h^{2}}+b \frac{u_{p+1}^{q}-u_{p-1}^{q}}{2 h}+c u_{p}^{q}, \\
p=1,2, \ldots, M-1, \quad q=0,1,2, \ldots, N-1 .
\end{gathered}
$$

Substitution of $u_{p}^{q}=G^{q} e^{i \beta p h}$ into the above difference equation leads to

$$
G-1=r a\left[e^{i \beta h}-2+e^{-i \beta h}\right]+b \frac{k}{2 h}\left[e^{i \beta h}-e^{-i \beta h}\right]+c k .
$$

Hence

$$
G \leq 1-4 r a \sin ^{2}\left(\frac{\beta h}{2}\right)+i\left(2 b \frac{k}{h}\right) \sin \left(\frac{\beta h}{2}\right) \cos \left(\frac{\beta h}{2}\right),
$$

satisfies the von Neumann condition if

$$
\left|1-4 r a \sin ^{2}\left(\frac{\beta h}{2}\right)+i\left(2 b \frac{k}{h}\right) \sin \left(\frac{\beta h}{2}\right) \cos \left(\frac{\beta h}{2}\right)\right| \leq 1 .
$$

After simplification above relation, we have

$$
r \leq \frac{2 a-b^{2} k \cos ^{2}\left(\frac{\beta h}{2}\right)}{4 a^{2} \sin ^{2}\left(\frac{\beta h}{2}\right)} .
$$

Therefore, the principle of frozen coefficients yields the following stability analysis based on criterion for the FTCS method to (2.5)

$$
r \leq \frac{1}{2 a(x, t)} .
$$




\section{Von Neumann stability analysis for parabolic inverse problem}

In this section, we give some theorems on the von Neumann stability analysis based on different schemes of finite difference for inverse coefficient problem (1.1) in one and two dimensional case.

Theorem 3.1. If we consider $\Omega=(0,1),\left(a_{j m}(x, t)\right)_{j, m=1}^{2}:=\left(\begin{array}{ll}a(t) & 0 \\ 0 & 0\end{array}\right), a(t)>0$, $d(t) \leq 0$ and $\left\{\varepsilon_{m}\right\}_{m=1}^{2}:=\{\varepsilon, 0\}$ in (1.1), then the FTCS method for (1.1) is stable whenever $r \leq \frac{1}{2 a(t)}$.

Proof. By using (1.1) and (1.2), we have

$$
a(t)=\frac{E^{\prime}(t)-\varepsilon F(t)-d(t) E(t)}{u_{x x}\left(x^{*}, t\right)} .
$$

Thus, problem (1.1) with some tedious manipulation yields the following problem

$$
\begin{cases}u_{t}(x, t)=\frac{E^{\prime}(t)-\varepsilon F(t)-d(t) E(t)}{u_{x x}\left(x^{*}, t\right)} u_{x x}(x, t) & +\varepsilon u_{x}(x, t)+d(t) u(x, t), \\ u(x, 0)=f(x), & x \in \Omega, \quad 0<t \leq T, \\ u(0, t)=g_{1}(t), & x \in \bar{\Omega}, \\ u(1, t)=g_{2}(t), & 0 \leq t \leq T,\end{cases}
$$

Let $v(x, t)=u_{x x}(x, t)$, then we have

$$
\begin{cases}v_{t}(x, t)=\frac{E^{\prime}(t)-\varepsilon F(t)-d(t) E(t)}{v\left(x^{*}, t\right)} v_{x x}(x, t) & +\varepsilon v_{x}(x, t)+d(t) v(x, t), \\ v(x, 0)=f^{\prime \prime}(x), & x \in \Omega, \quad 0<t \leq T, \\ v(0, t)=\frac{g_{1}^{\prime}(t)-d(t) g_{1}(t)}{E^{\prime}(t)-\varepsilon F(t)-d(t) E(t)} v\left(x^{*}, t\right), & 0 \leq t \leq T, \\ v(1, t)=\frac{g_{2}^{\prime}(t)-d(t) g_{2}(t)}{E^{\prime}(t)-\varepsilon F(t)-d(t) E(t)} v\left(x^{*}, t\right), & 0 \leq t \leq T .\end{cases}
$$

This problem is nonlinear, therefore if we give the von Neumann stability analysis, then we have to reduce it to a linear form. For this purpose, we assume $w$ is another solution of (3.3) that is nearby to $v$. Their difference satisfies

$$
\begin{aligned}
\left(v_{t}-w_{t}\right)(x, t) & =\left(\frac{E^{\prime}(t)-\varepsilon F(t)-d(t) E(t)}{v\left(x^{*}, t\right)} v_{x x}(x, t)+\varepsilon v_{x}(x, t)+d(t) v(x, t)\right) \\
& -\left(\frac{E^{\prime}(t)-\varepsilon F(t)-d(t) E(t)}{w\left(x^{*}, t\right)} w_{x x}(x, t)+\varepsilon w_{x}(x, t)+d(t) w(x, t)\right) .
\end{aligned}
$$

We linearized (3.4) about $w$. Define

$$
f\left(v, v_{x}, v_{x x}\right)=\frac{E^{\prime}(t)-\varepsilon F(t)-d(t) E(t)}{v\left(x^{*}, t\right)} v_{x x}(x, t)+\varepsilon v_{x}(x, t)+d(t) v(x, t) .
$$

By using an equivalent form of the chain rule for function $f\left(v, v_{x}, v_{x x}\right)$, we have

$$
d f\left(v, v_{x}, v_{x x}\right)=f_{v} d v+f_{v_{x}} d v_{x}+f_{v_{x x}} d v_{x x} .
$$

If we replace differentials by small but finite increase, then (3.5) will be

$$
\Delta f\left(v, v_{x}, v_{x x}\right) \simeq f_{v} \Delta v+f_{v_{x}} \Delta v_{x}+f_{v_{x x}} \Delta v_{x x},
$$


where

$$
\begin{aligned}
& \Delta v=v-w, \Delta v_{x}=v_{x}-w_{x}, \Delta v_{x x}=v_{x x}-w_{x x}, \\
& \Delta f\left(v, v_{x}, v_{x x}\right)=f\left(v, v_{x}, v_{x x}\right)-f\left(w, w_{x}, w_{x x}\right) .
\end{aligned}
$$

Based on (3.6) and (3.7), the right hand side of (3.5) is as follows:

$$
\begin{aligned}
& \left(d(t)-\frac{E^{\prime}(t)-\varepsilon F(t)-d(t) E(t)}{v^{2}\left(x^{*}, t\right)} v_{x x}\right) \Delta v(x, t) \\
& +\varepsilon \Delta v_{x}(x, t)+\frac{E^{\prime}(t)-\varepsilon F(t)-d(t) E(t)}{v\left(x^{*}, t\right)} \Delta v_{x x}(x, t) .
\end{aligned}
$$

On the other hand, we know that

$$
v_{t}(x, t)=f\left(v, v_{x}, v_{x x}\right),
$$

so, we have

$$
\begin{aligned}
\Delta v_{t}(x, t) & \approx \frac{E^{\prime}(t)-\varepsilon F(t)-d(t) E(t)}{v\left(x^{*}, t\right)} \Delta v_{x x}(x, t) \\
& +\varepsilon \Delta v_{x}(x, t)+\left(d(t)-\frac{E^{\prime}(t)-\varepsilon F(t)-d(t) E(t)}{v^{2}\left(x^{*}, t\right)} v_{x x}(x, t)\right) \Delta v(x, t) .
\end{aligned}
$$

Equation (3.9) is linearized of (3.3) and dependent on $\Delta v$. If we compare (3.9) with equation $u_{t}=a(x, t) u_{x x}(x, t)+b(x, t) u_{x}(x, t)+c(x, t) u(x, t)$, we have

$$
\begin{aligned}
& a(x, t):=\frac{E^{\prime}(t)-\varepsilon F(t)-d(t) E(t)}{v\left(x^{*}, t\right)}, b(x, t):=\varepsilon, \\
& c(x, t):=d(t)-\frac{E^{\prime}(t)-\varepsilon F(t)-d(t) E(t)}{v^{2}\left(x^{*}, t\right)} v_{x x}(x, t) .
\end{aligned}
$$

According to the principle of frozen coefficients, the necessary condition for stability is

$$
r \leq \frac{v\left(x^{*}, t\right)}{2\left(E^{\prime}(t)-\varepsilon F(t)-d(t) E(t)\right)} .
$$

If we replace $v\left(x^{*}, t\right)$ by $u_{x x}\left(x^{*}, t\right)$ and using $(3.1)$, then the proof is complete.

Theorem 3.2. If we consider $\Omega=(0,1),\left(a_{j m}(x, t)\right)_{j, m=1}^{2}:=\left(\begin{array}{ll}a(t) & 0 \\ 0 & 0\end{array}\right), a(t)>0$, $d(t) \leq 0$ and $\left\{\varepsilon_{m}\right\}_{m=1}^{2}:=\{\varepsilon, 0\}$ in (1.1), then the BTCS method for (1.1) is unconditionally stable for all $r>0$.

Proof. According to the proof of Theorem 3.1, we obtain an equation by the form

$$
u_{t}(x, t)=a(x, t) u_{x x}(x, t)+b(x, t) u_{x}(x, t)+c(x, t) u(x, t) .
$$

Assume coefficients $a(x, t), b(x, t)$ and $c(x, t)$ are freezed. By applying the BTCS for (3.10), we have

$$
\begin{gathered}
\frac{u_{p}^{q+1}-u_{p}^{q}}{k}=a(x, t) \frac{u_{p+1}^{q+1}-2 u_{p}^{q+1}+u_{p-1}^{q+1}}{h^{2}}+b(x, t) \frac{u_{p+1}^{q+1}-u_{p-1}^{q+1}}{2 h}+c(x, t) u_{p}^{q+1}, \\
p=1,2, \ldots, M-1, q=0,1,2, \ldots, N-1 .
\end{gathered}
$$

We apply von Neumann stability analysis for (3.11), so

$$
\begin{aligned}
& G^{q+1} e^{i \beta p h}-G^{q} e^{i \beta p h}= \\
& r a(x, t)\left[G^{q+1} e^{i \beta(p+1) h}-2 G^{q+1} e^{i \beta p h}+G^{q+1} e^{i \beta(p-1) h}\right] \\
& +b(x, t) \frac{k}{2 h}\left[G^{q+1} e^{i \beta(p+1) h}-G^{q+1} e^{i \beta(p-1) h}\right]+c(x, t) k G^{q+1} e^{i \beta p h} .
\end{aligned}
$$


After simplification, we have

$$
\begin{aligned}
G-1 & =r a(x, t) G\left[e^{i \beta h}-2+e^{-i \beta h}\right]+b(x, t) \frac{k}{2 h} G\left[e^{i \beta h}-e^{-i \beta h}\right]+c(x, t) k G \\
& =r a(x, t) G\left[-4 \sin ^{2}\left(\frac{\beta h}{2}\right)\right]+b(x, t) \frac{k}{h} G[i \sin (\beta h)]+c(x, t) k G .
\end{aligned}
$$

Thus

$$
G\left[1+4 r a(x, t) \sin ^{2}\left(\frac{\beta h}{2}\right)-i b(x, t) \frac{k}{h} \sin (\beta h)-c(x, t) k\right]=1 .
$$

From above equation, we have

$$
G=\frac{1}{1+4 r a(x, t) \sin ^{2}\left(\frac{\beta h}{2}\right)-i b(x, t) \frac{k}{h} \sin (\beta h)-c(x, t) k} .
$$

So for all $r>0$, we have

$$
|G| \leq 1
$$

We emphasize that if $G$ satisfies $|G| \leq 1$, then we take $c(x, t) \leq 0$.

Theorem 3.3. If we consider $\Omega=(0,1),\left(a_{j m}(x, t)\right)_{j, m=1}^{2}:=\left(\begin{array}{ll}a(t) & 0 \\ 0 & 0\end{array}\right), a(t)>0$, $c(x, t) \leq 0, d(t) \leq 0$ and $\left\{\varepsilon_{m}\right\}_{m=1}^{2}:=\{\varepsilon, 0\}$ in (1.1), then the CN method for (1.1) is unconditionally stable for all $r>0$.

Proof. As seen in the proof of Theorem 3.1, we obtain an equation like (2.5). By implementation of Crank Nicolson method for (2.5), we have

$$
\begin{aligned}
\frac{u_{p}^{q+1}-u_{p}^{q}}{k} & =\frac{1}{2} a(x, t)\left[\frac{u_{p+1}^{q+1}-2 u_{p}^{q+1}+u_{p-1}^{q+1}}{h^{2}}+\frac{u_{p+1}^{q}-2 u_{p}^{q}+u_{p-1}^{q}}{h^{2}}\right] \\
& +\frac{1}{2} b(x, t)\left[\frac{u_{p+1}^{q+1}-u_{p-1}^{q+1}}{2 h}+\frac{u_{p+1}^{q}-u_{p-1}^{q}}{2 h}\right]+c(x, t) u_{p}^{q},
\end{aligned}
$$

for $p=1,2, \ldots, M-1$, and $q=0,1,2, \ldots, N-1$.

If we put $u_{p}^{q}:=G^{q} e^{i \beta p h}$ in (3.12), we have

$$
\begin{aligned}
& G-1=\frac{1}{2} r a(x, t)(2 \cos (\beta h)-2)(G+1)+i\left(\frac{k}{2 h}\right) b(x, t) \sin (\beta h)(G+1) \\
& +c(x, t) k=\left(-2 r a(x, t) \sin ^{2}\left(\frac{\beta h}{2}\right)+i\left(\frac{k}{2 h}\right) b(x, t) \sin (\beta h)\right)(G+1)+c(x, t) k .
\end{aligned}
$$

We put $A=-2 r a(x, t) \sin ^{2}\left(\frac{\beta h}{2}\right)+i\left(\frac{k}{2 h}\right) b(x, t) \sin (\beta h)$, so for all $r>0$, we have

$$
G=1+A(G+1)+c(x, t) k \leq 1+A(G+1),
$$

and

$$
|G| \leq\left|\frac{1+A}{1-A}\right|
$$

where $|1-A| \geq 1$ and $\left|\frac{1+A}{1-A}\right| \leq 1$. To prove these inequalities, we have

$$
|1-A|^{2}=\left(1+2 r a(x, t) \sin ^{2}\left(\frac{\beta h}{2}\right)\right)^{2}+\frac{1}{4} k r b^{2}(x, t) \sin ^{2}(\beta h) .
$$

It is clear that $|1-A|^{2} \geq 1$ so $|1-A| \geq 1$.

On the other hand

$$
\begin{aligned}
\left|\frac{1+A}{1-A}\right|^{2} & =\left|\frac{1-2 r a(x, t) \sin ^{2}\left(\frac{\beta h}{2}\right)+i b(x, t) \frac{k}{2 h} \sin (\beta h)}{1+2 r a(x, t) \sin ^{2}\left(\frac{\beta h}{2}\right)-i b(x, t) \frac{k}{2 h} \sin (\beta h)}\right|^{2} \\
& =\frac{\left(1-2 r a(x, t) \sin ^{2}\left(\frac{\beta h}{2}\right)\right)^{2}+r b^{2}(x, t) \frac{k}{4} \sin ^{2}(\beta h)}{\left(1+2 r a(x, t) \sin ^{2}\left(\frac{\beta h}{2}\right)^{2}\right)+r b^{2}(x, t) \frac{k}{4} \sin ^{2}(\beta h)} .
\end{aligned}
$$


Since numerator in (3.13) is less than denominator, therefore $\left|\frac{1+A}{1-A}\right| \leq 1$, hence (3.12) for all $r>0$ is unconditionally stable.

Theorem 3.4. Let $\left(a_{j m}(x, t)\right)_{j, m=1}^{2}$ be a symmetric, positive definite $2 \times 2$ matrix. For the following problem

$$
\left\{\begin{array}{lll}
u_{t}(x, t)=\sum_{j, m=1}^{2} a_{j m}(x, t) \frac{\partial^{2} u(x, t)}{\partial x_{j} \partial x_{m}} & \\
+\sum_{m=1}^{2} \varepsilon_{m} u_{x_{m}}(x, t)+d(t) u(x, t), & x \in \Omega \subseteq \mathbb{R}^{2}, \quad 0<t \leq T, \\
u(x, 0)=f(x), & x \in \bar{\Omega}, \\
u(x, t)=g(t), & x \in \partial \Omega, \quad 0 \leq t \leq T .
\end{array}\right.
$$

Subject to extra measurements

$$
\begin{aligned}
& u\left(x_{1}^{*}, x_{2}^{*}, t\right)=E(t), \quad u_{x_{1}}\left(x_{1}^{*}, x_{2}^{*}, t\right)=F_{1}(t), \\
& u_{x_{2}}\left(x_{1}^{*}, x_{2}^{*}, t\right)=F_{2}(t), \quad 0 \leq t \leq T, \quad x^{*} \in \Omega .
\end{aligned}
$$

If we assume the following conditions, then (3.14) subject to (3.15) has a unique solution and $u \in C^{4,2}(\Omega \times[0, T]):$

$i$ - $f(x) \in C^{4+\alpha}(\Omega), \Delta f(x)>0$ and $\Delta^{2} f\left(x^{*}\right)>0$, and $\Delta^{2} f(x)>0$, on $\Omega$.

$i i-g(t), E(t), F_{j}(t) \in C^{2+\frac{\alpha}{2}}([0, T]), F_{j}(t), E^{\prime}(t)>0, d(t) \leq 0, \quad 0<\frac{g_{i}(t)}{E^{\prime}(t)}<1$,

$\left(\frac{g_{i}(t)}{E^{\prime}(t)}\right)^{\prime}>0$ on $[0, T]$ and here $0 \leq \varepsilon_{m}<<0$ and $j \in\{1,2\}, i \in\{1,2,3,4\}$.

Moreover, if we apply the finite difference methods then

1- The FTCS method is stable whenever $r \leq \frac{1}{\left\|\left(a_{j m}(x, t)\right)_{j, m=1}^{2}\right\|_{L_{\infty}}}$.

2- The BTCS method is unconditionally stable for all $r>0$.

3- The CN method is unconditionally stable for all $r>0$.

Where $r=\frac{k}{\|h\|_{L_{\infty}}^{2}}, h=\left(h_{1}, h_{2}\right)$ the mesh-width in $x_{1}$ and $x_{2}$, respectively. Also, $k$ is the time step size.

Proof. The existence and uniqueness of the solutions to similar of this problem are discussed in [7]. It is shown [6] that the above conditions lead to [4] an existence and uniqueness theorem. For the numerical solution of this problem by finite differences one introduces a grid of mesh points $\left(x_{1}, x_{2}, t\right)=\left(x_{1}^{i}, x_{2}^{j}, t_{n}\right)$. Here $x_{1}^{i}=i h_{1}, x_{2}^{j}=j h_{2}$, and $t_{n}=n k$, where $i, j$ and $n$ are integers, $n \geq 0$. Define $h_{1}$ and $h_{2}$ as the mesh width in $x_{1}$, $x_{2}$ direction, respectively and $k$ as the time step. One then seeks an approximate solution $u_{i j}^{n}$ at these mesh points, determined by an equation obtained by replacing the derivatives in (3.14) by difference quotients. For functions defined on the grid we introduce thus the forward, backward and Crank Nicolson methods. By improving the above theorems, we conclude the results.

Table 1. $L_{\infty}$ norm for error of $u$ with different methods when $r=0.0001$.

\begin{tabular}{cccc}
\hline$t$ & FTCS & BTCS & CN \\
\hline 0.2 & $1.7 \times 10^{-3}$ & $1.3 \times 10^{-5}$ & $2.8 \times 10^{-5}$ \\
0.4 & $3.5 \times 10^{-3}$ & $4.6 \times 10^{-5}$ & $2.4 \times 10^{-6}$ \\
0.6 & $4.1 \times 10^{-3}$ & $6.7 \times 10^{-5}$ & $4.8 \times 10^{-5}$ \\
0.8 & $3.9 \times 10^{-3}$ & $7.9 \times 10^{-6}$ & $3.6 \times 10^{-5}$ \\
\hline
\end{tabular}

\section{Numerical results}

In this section, in order to demonstrate the efficiency of our theorems, we apply the proposed methods that are FTCS, BTCS and CN to solve one example. To show the accuracy of the methods, we use maximum absolute error. 
Table 2. $L_{\infty}$ norm for error of $u$ with different methods when $r=2$.

\begin{tabular}{cccc}
\hline$t$ & FTCS & BTCS & CN \\
\hline 0.2 & $4.1 \times 10^{-3}$ & $7.1 \times 10^{-5}$ & $1.0 \times 10^{-5}$ \\
0.4 & $1.1 \times 10^{-2}$ & $4.8 \times 10^{-5}$ & $1.3 \times 10^{-6}$ \\
0.6 & $5.3 \times 10^{-3}$ & $1.6 \times 10^{-5}$ & $2.8 \times 10^{-5}$ \\
0.8 & $2.6 \times 10^{-2}$ & $2.3 \times 10^{-6}$ & $5.1 \times 10^{-6}$ \\
\hline
\end{tabular}

Example 4.1. We consider inverse problem (1.1) with

$$
\varepsilon=\left(\varepsilon_{1}, \varepsilon_{2}\right)^{T}, \quad \varepsilon_{1}=\varepsilon_{2}=0.1, \quad d(t)= \begin{cases}-1000 t^{2}, & 0<t \leq \frac{T}{2}, \\ 0, & \frac{T}{2}<t \leq T,\end{cases}
$$

the initial condition

$$
u\left(x_{1}, x_{2}, 0\right)=\exp \left(x_{1}+x_{2}\right), \quad \Omega=[0,1] \times[0,1],
$$

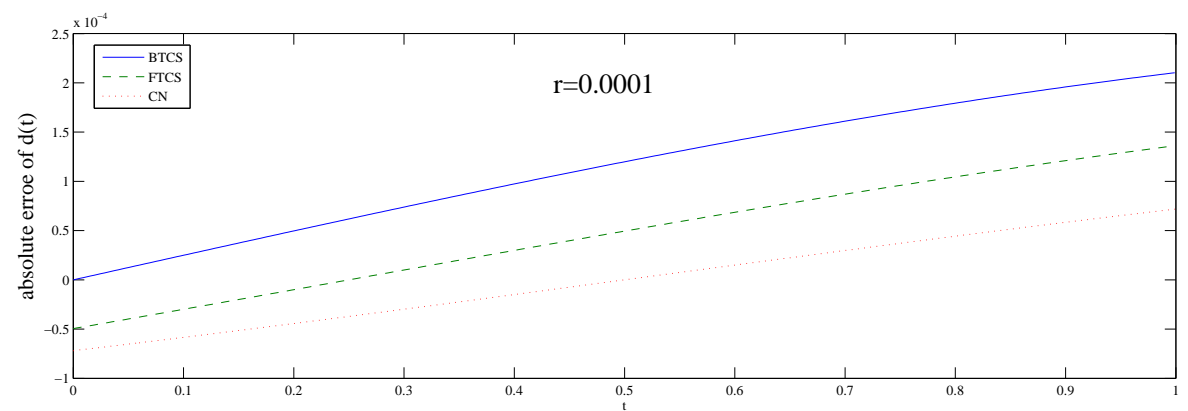

Fig. 1. The absolute error of $d(t)$ with $L_{\infty}$ norm for $r=0.0001$ related to time .

and the boundary conditions

$$
\begin{aligned}
& u(0,0, t)=g_{1}(t)=\exp (t), \\
& u(0,1, t)=g_{2}(t)=\exp (1+t), \\
& u(1,0, t)=g_{3}(t)=\exp (1+t), \\
& u(1,1, t)=g_{4}(t)=\exp (2+t) .
\end{aligned}
$$

We choose $x_{1}^{*}=x_{2}^{*}=0.25$, Hence

$$
E(t)=F_{1}(t)=F_{2}(t)=\exp (0.5+t) .
$$

Note that the exact solution of this problem is

$$
u\left(x_{1}, x_{2}, t\right)=\exp \left(x_{1}+x_{2}+t\right), \quad a_{j, m}(t)=\frac{1}{4}(1-2 \varepsilon-d(t)), \quad j, m=1,2 .
$$

According to Theorem 3.4, we emphasize that $d(t)$ must be non-positive. We solve this problem at $T=1$. Table 1 shows absolute error for $u$ with $k=0.01$ and $h_{1}=h_{2}=0.001$ and hence $r=0.0001$. Fig 1 shows the plot of error $d(t)$ for $r=0.0001$. We observe that FTCS, BTCS and CN methods are stable for all positive value of $r \leq \frac{1}{\left\|a_{j m}\left(x_{1}, x_{2}, t\right)\right\|_{L_{\infty}}}$. The results obtained for $u$ with $k=0.01, h_{1}=h_{2}=0.07$ and hence $r=2$ are presented in Table 2. Also, Table 3 gives the absolute error of $u$ with $k=0.01, h_{1}=h_{2}=0.0025$ and $r=40$. Figs 2, 3 demonstrate the plot of error $d(t)$, for $r=2$ and $r=4$, respectively. In Figs 2, 3 and Tables 2, 3, we observe that for large values of $r$, BTCS and CN are stable. On the other hand, the absolute error obtained by applying FTCS is recorded 


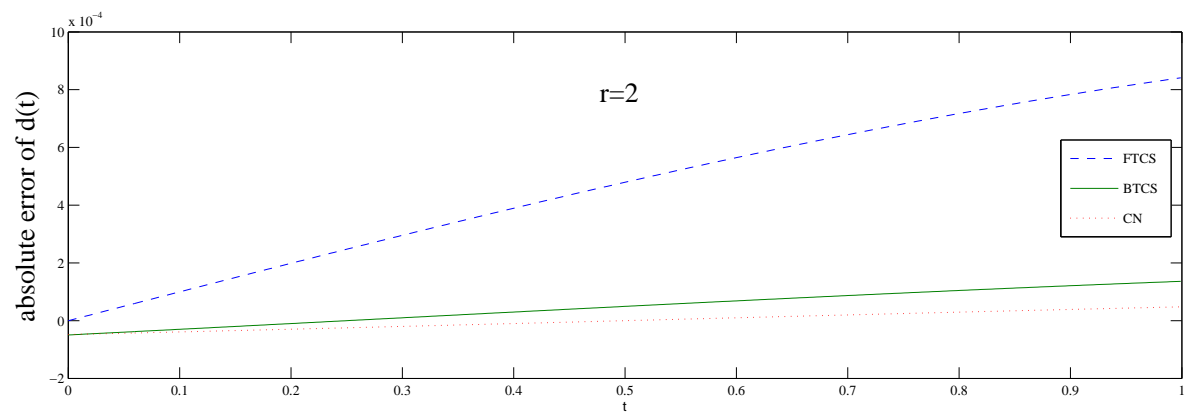

Fig. 2. The absolute error of $d(t)$ with $L_{\infty}$ norm for $r=2$ related to time .

Table 3. $L_{\infty}$ norm for error of $u$ with different methods when $r=40$.

\begin{tabular}{cccc}
\hline$t$ & FTCS & BTCS & CN \\
\hline 0.2 & $9.1 \times 10^{-2}$ & $2.3 \times 10^{-5}$ & $4.6 \times 10^{-5}$ \\
0.4 & $7.3 \times 10^{-1}$ & $2.8 \times 10^{-6}$ & $1.7 \times 10^{-5}$ \\
0.6 & $8.1 \times 10^{-2}$ & $3.7 \times 10^{-6}$ & $3.7 \times 10^{-6}$ \\
0.8 & $9.9 \times 10^{-2}$ & $5.9 \times 10^{-5}$ & $5.6 \times 10^{-6}$ \\
\hline
\end{tabular}

in the tables. These results of three methods clearly indicate that the value of $r$ is very important for FTCS and this method is valid only for $0<r \leq \frac{1}{\left\|\left(a_{j k}\left(x_{1}, x_{2}, t\right)\right)_{j, m=1}^{2}\right\|_{L_{\infty}}}$.

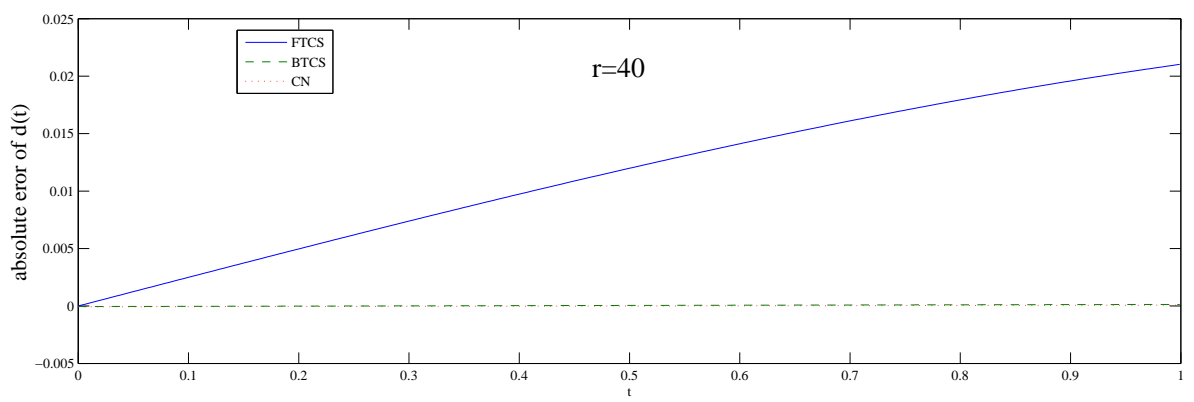

Fig. 3. The absolute error of $d(t)$ with $L_{\infty}$ norm for $r=40$ related to time .

\section{Conclusion}

In this paper, we proposed three numerical schemes for solving an inverse problem with extra measurements. We show that the FTCS scheme is conditional method for solving an inverse problem. But BTCS and CN methods are suitable schemes because they are unconditional method. We justified this advantage of the stability analysis versus the numerical method with an example.

Acknowledgment. The authors would like to express their thankfulness to the referees for valuable comments and Professor George Dulikravich whose constructive comments improved the quality of this paper.

\section{References}

[1] M. Asadzadeh, D. Rostamy and F. Zabihi, A posteriori error estimates for the solutions of a coupled wave system, J. Math. Sci. 175 (2), 228-248, 2011.

[2] M. Asadzadeh, D. Rostamy and F. Zabihi, Discontinuous Galerkin and multiscale variational schemes for a coupled damped nonlinear system of Schrödinger equations, Numer. Methods Partial Differential Equations, 29 (5), 1-34, 2013. 
[3] C. Ashyralyyev and M. Dedeturk, A finite difference method for the inverse elliptic problem with the dirichlet condition, Contemp. Anal. Appl. Math. 1, 132-155, 2013.

[4] H. Azari, W. Allegretto, Y. Lin and S. Zhang, Numerical procedures for recovering a time dependent coefficient in a parabolic differential equation, Dyn. Contin. Discrete Impuls. Syst. Ser. B Appl. Algorithms, 11, 181-199, 2004.

[5] J. Blazek, Computational Fluid Dynamics: Principles and Applications, Elsevier, 2005.

[6] J.R. Cannon, Determination of an unknown coefficient in a parabolic differential equation, Duke. Math. J. 30, 313-323, 1963.

[7] J.R. Cannon and H.M. Yin, Numerical solutions of some parabolic inverse problems, Numer. Methods Partial Differential Equations, 2, 177-191, 1990.

[8] M. Dehghan, Identification of a time-dependent coefficient in a partial differential equation subject to an extra measurement, Numer. Methods Partial Differential Equations, 21, 611-622, 2005.

[9] S.K. Godunov and V.S. Ryabenkii, Difference Schemes: An Introduction to the Underlying Theory, Studies in Mathematics and Its Applications, 1987.

[10] P. Jonas and A.K. Louis, Approximate inverse for a one-dimensional inverse heat conduction problem, Inverse Problems, 16, 175-185, 2000.

[11] B.F. Jones, Various methods for finding unknown coefficients in a parabolic differential equations,Comm. Pure Appl. Math. 16, 33-44, 1963.

[12] A. Kirsch, An Introduction to the Mathematical Theory of Inverse Problems, Springer, New York, 1999.

[13] M. Lakestani and M. Dehghan, The use of chebyshev cardinal functions for the solution of a partial differential equation with an unknown time-dependent coefficient subject to an extra measurement, J. Comput. Appl. Math. 235, 669-678, 2010.

[14] D. Lesnic and L. Elliot, The decomposition approach to inverse heat conduction, J. Math. Anal. Appl. 232, 82-98, 1999.

[15] Y. Lin, Analytical and numerical solutions for a class of nonlocal nonlinear parabolic differential equations, SIAM J. Math. Anal. 25 (6), 1577-1594, 1994.

[16] J.A. Macbain, Inversion theory for a parameterized diffusion problem, SIAM J. Appl. Math. 18, 1386-1391, 1987.

[17] A.R. Mitchell and D.F. Griffiths, The finite difference method in partial differential equations, John Wiley \& Sons, Chichester, 1980.

[18] A. Mohebbi and M. Dehghan, High-order scheme for determination of a control parameter in an inverse problem from the over-specified data, Commun. Comput. Phys. 181, 1947-1954, 2010.

[19] D. Rostamy, The new streamline diffusion for 3D Coupled Schrodinger equations with a cross-phase modulation, ANZIAM J. 55, 51-78, 2013.

[20] D. Rostamy, F. Zabihi, A. Niroomand and A. Mollazeynal, New Finite Element Method for Solving a Wave Equation with a Nonlocal Conservation Condition, Transport Theory Statist. Phys. 42, 41-62, 2013.

[21] D. Rostamy and A. Abdollahi, Gegenbauer Cardinal Functions for the Inverse Source Parabolic Problem with a Time-Fractional Diffusion Equation, Transport Theory Statist. Phys. 46, 307-329, 2017.

[22] A. Samarskii and A. Vabishchevich, Numerical methods for solving inverse problems of mathematical physics, Walter de Gruyter, Berlin, 2007.

[23] J.C. Strikwerda, Finite difference schemes and partial differential equations, Wadsworth \& Brooks/ Cole, California, 1989.

[24] A. Tikhonov and V. Arsenin, Solution methods for Ill-posed problems, Nauka, Moscow, 1986.

[25] A. Tveito and R. Winther, Introduction to Partial Differential Equations, SpringerVerlag, New York, Inc., 1998. 
[26] C. Udriste, V. Arsinte and C. Cipu, Von Neumann analysis of linearized discrete Tzitzeica PDE, Balkan J. Geom. Appl. 15 (2), 100-112, 2010.

[27] A. Vabishchevich and A. Samarskii, Additive schemes for mathematical-physics problems, Nauka, Moscow, 1999.

[28] S. Wang and Y. Lin, A finite difference solution to an inverse problem determining a control function in a parabolic partial differential equations, Inverse Problems, 5, 631-640, 1989.

[29] L. Yan, C.L. Fu and F.L. Yang, The method of fundamental solutions for the inverse heat source problem, Eng. Anal. Boundary Elem. 32, 216-222, 2008. 